\title{
NOTE ON A $q$-IDENTITY
}

\author{
W. A. AL-SALAM
}

Fjeldstad [4] proved the identity

$$
\begin{aligned}
\sum_{s=0}^{2 m}(-1)^{s}\left(\begin{array}{c}
2 m \\
s
\end{array}\right) & \left(\begin{array}{c}
2 n \\
n-m+s
\end{array}\right)\left(\begin{array}{c}
2 p \\
p-m+s
\end{array}\right) \\
& =(-1)^{m} \frac{(m+n+p) !(2 m) !(2 n) !(2 p) !}{(m+n) !(m+p) !(n+p) ! m ! n ! p !}
\end{aligned}
$$

and later Carlitz [2] gave its $q$-analog

$$
\begin{aligned}
\sum_{s=0}^{2 m}(-1)^{s}\left[\begin{array}{c}
2 m \\
s
\end{array}\right]\left[\begin{array}{c}
2 n \\
n-m+s
\end{array}\right]\left[\begin{array}{c}
2 p \\
p-m+s
\end{array}\right] q^{\frac{1}{\left\{\left(3(s-m)^{2}+(s-m)\right\}\right.}} \\
=(-1)^{m} \frac{[m+n+p] ![2 m] ![2 n] ![2 p] !}{[m+n] ![m+p] ![n+p] ![m] ![n] ![p] !} .
\end{aligned}
$$

Carlitz obtained (2) by specializing Jackson's formula

$$
\sum_{s=0}^{2 m}(-1)^{s}\left[\begin{array}{c}
2 m \\
s
\end{array}\right](a)_{s}(b)_{s}(a)_{2 m-s}(b)_{2 m-s} q^{\frac{1}{2(2(2 m-s+1)}}=(a)_{m}(b)_{m}\left(q^{m+1}\right)_{m}\left(a b q^{m}\right)_{m}
$$

where

$$
\begin{gathered}
(a)_{s}=(1-a)(1-a q) \ldots\left(1-a q^{s-1}\right), \quad(a)_{0}=1, \\
{\left[\begin{array}{l}
n \\
s
\end{array}\right]=\frac{(q)_{n}}{(q)_{s}(q)_{n-s}}, \quad[n] !=(q)_{n} .}
\end{gathered}
$$

Hence it might be of interest to see what is implied by Jackson's more general formula [5]

$$
\begin{aligned}
& \sum_{s=0}^{2 m}(-1)^{s}\left[\begin{array}{c}
2 m \\
s
\end{array}\right](a)_{s}(b)_{s}(c)_{s}(d)_{s}(a)_{2 m-s}(b)_{2 m-s}(c)_{2 m-s}(d)_{2 m-s} q^{\frac{1}{2} s(6 m-3 s+1)} \\
& =(-1)^{m}(a)_{m}(b)_{m}(c)_{m}(d)_{m}\left(q^{m+1}\right)_{m}\left(a b q^{m}\right)_{m}\left(b c q^{m}\right)_{m}\left(a c q^{m}\right)_{m} d^{m} q^{\frac{1}{2} m(3 m-1)}
\end{aligned}
$$

where $a b c d=q^{1-3 n}$.

Received July 15, 1957. 
Put in (3)

$$
a=q^{-m-p_{1}}, \quad b=q^{-m-p_{2}}, \quad c=q^{-m-p_{3}}, \quad \text { and } \quad d=q^{1+p},
$$

where

Hence (3) reduces to

$$
p=p_{1}+p_{2}+p_{3}
$$

(4) $\sum_{s=0}^{2 m}(-1)^{s}\left[\begin{array}{c}2 m \\ s\end{array}\right]\left[\begin{array}{c}2 p_{1} \\ p_{1}-m+s\end{array}\right]\left[\begin{array}{c}2 p_{2} \\ p_{2}-m+s\end{array}\right]\left[\begin{array}{c}2 p_{3} \\ p_{3}-m+s\end{array}\right]\left[\begin{array}{c}p+s \\ s\end{array}\right]\left[\begin{array}{c}p+2 m \\ s\end{array}\right]^{-1} q^{\frac{1}{2} s(3 s-6 m+1)}$

$$
\begin{gathered}
=(-1)^{m} \frac{\left[2 p_{1}\right] !\left[2 p_{2}\right] !\left[2 p_{3}\right] ![m+p] !\left(q^{m+1}\right)_{m}\left(q^{p+1}\right)^{m}}{\left[m+p_{1}\right] !\left[m+p_{2}\right] !\left[m+p_{3}\right] ![p+2 m] !\left[p_{1}\right] !\left[p_{2}\right] !\left[p_{3}\right] !} \\
\cdot \frac{\left[m+p_{1}+p_{2}\right] !\left[m+p_{1}+p_{3}\right] !\left[m+p_{2}+p_{3}\right] !}{\left[p_{1}+p_{2}\right] !\left[p_{1}+p_{3}\right] !\left[p_{2}+p_{3}\right] !} q^{-\frac{1}{2} m(2 p+3 m+1)}
\end{gathered}
$$

In particular if $p_{1}=p_{2}=p_{3}=m$, (4) reduces to

$$
\begin{aligned}
\sum_{s=0}^{2 m}(-1)^{s}\left[\begin{array}{c}
2 m \\
s
\end{array}\right]^{4}\left[\begin{array}{c}
3 m+s \\
s
\end{array}\right]\left[\begin{array}{c}
5 m \\
s
\end{array}\right]^{-1} q^{\frac{1}{2} s(3 s-6 m+1)} \\
=(-1)^{m}\left[\begin{array}{c}
3 m \\
m
\end{array}\right]^{3} \frac{[4 m] !\left(q^{m+1}\right)_{m}\left(q^{p+1}\right)^{m}}{[5 m] !} q^{-\frac{1}{2} m(9 m+1)} .
\end{aligned}
$$

Now if we take the limit as $q \rightarrow 1$, formulas (4) and (5) reduce respectively to

(6)

$$
\begin{gathered}
\sum_{s=0}^{2 m}(-1)^{s}\left(\begin{array}{c}
2 m \\
s
\end{array}\right)\left(\begin{array}{c}
2 p_{1} \\
p_{1}-m+s
\end{array}\right)\left(\begin{array}{c}
2 p_{2} \\
p_{2}-m+s
\end{array}\right)\left(\begin{array}{c}
2 p_{3} \\
p_{3}-m+s
\end{array}\right)\left(\begin{array}{c}
p+s \\
s
\end{array}\right)\left(\begin{array}{c}
p+2 m \\
s
\end{array}\right)^{-1} \\
=(-1)^{m} \frac{\left(2 p_{1}\right) !\left(2 p_{2}\right) !\left(2 p_{3}\right) !(m+p) !(2 m) !\left(m+p_{1}+p_{2}\right) !}{\left(m+p_{1}\right) !\left(m+p_{2}\right) !\left(m+p_{3}\right) !(p+2 m) ! p_{1} ! p_{2} ! p_{3} !} \\
\cdot \frac{\left(m+p_{1}+p_{3}\right) !\left(m+p_{2}+p_{3}\right) !}{\left(p_{1}+p_{2}\right) !\left(p_{1}+p_{3}\right) !\left(p_{2}+p_{3}\right) !}
\end{gathered}
$$

$$
\sum_{s=0}^{2 m}(-1)^{s}\left(\begin{array}{c}
2 m \\
s
\end{array}\right)^{4}\left(\begin{array}{c}
3 m+s \\
s
\end{array}\right)\left(\begin{array}{c}
5 m \\
s
\end{array}\right)^{-1}=(-1)^{m}\left(\begin{array}{c}
3 m \\
m
\end{array}\right)^{3} \frac{(4 m) !(2 m) !}{(5 m) ! m !}
$$

Finally we remark that if we let $p_{3} \rightarrow \infty$, then formula (6) reduces to Fjeldstad's formula (1), and (4) reduces to (2).

It is not clear whether (6) follows from Dougall's theorem (see [3] and $[1, \S 4.3])$. 


\section{REFERENCES}

1. W. N. Bailey, Generalized hypergeometric series (Cambridge Tracts in Mathematics and Mathematical Physics 32), London, 1935.

2. L. Carlitz, Note on a q-identity, Math. Scand. 3 (1955), 281-282.

3. J. Dougall, On Vandermonde's theorem and some more general expansions, Proc. Edinburgh Math. Soc. 25 (1907), 114-132.

4. J. E. Fjeldstad, A generalization of Dixon's formula, Math. Scand. 2 (1954), 46-48.

5. F. H. Jackson, Certain q-identities, Quart. J. Math. Oxford Ser. 12 (1941), 167-172.

DUKE UNIVERSTY, DURHAM, N.C., U.S.A. 